\title{
Zum Pronomen es bei Wetterverben im Jiddischen im Vergleich zu deutschen und polnischen Entsprechungen
}

\section{Einleitung}

In diesem Aufsatz gehe ich der Frage nach, welche syntaktischen Merkmale das Pronomen es in den jiddischen Sätzen mit Wetterverben aufweist und inwieweit diese Merkmale den Merkmalen der deutschen und der polnischen Sprache als zwei Kontaktsprachen in der Entwicklung des Jiddischen entsprechen. Anhand syntaktischer Tests wird untersucht, in wie vielen unterschiedlichen Positionen im Satz das Pronomen es vorkommen kann, in welchen Konfigurationen seine lexikalische Realisierung entfallen kann und in welchen sie obligatorisch ist.

Der Beitrag ist im theoretischen Rahmen des Prinzipien- und Parameter-Modells (PPT) (Chomsky 1981, Chomsky/Lasnik 1993) und des Minimalistischen Programms (MP) (Chomsky 1995) angesiedelt. Die Idee ist, dass für die Strukturierungsmöglichkeiten in den verschiedenen Sprachen ein Merkmals-Checking-Prozess (die Merkmalüberprüfung) verantwortlich ist. „Der Prozess der Merkmalüberprüfung besteht darin, dass ein Element mit zu überprüfenden Merkmalen in die Überprüfungsdomäne eines für die Überprüfung relevanten Kopfes bewegt wird" (Grewendorf 2002:160). Ausschlaggebend für das Checken sind somit die parametrisierten Eigenschaften funktionaler Kategorien.

In Bezug auf die zu überprüfenden Merkmale wird dafür argumentiert, dass bei den es-Elementen im Jiddischen und im Deutschen zwischen dem Argumenten-es und den Expletiva ${ }^{1}$ unterschieden werden muss.

${ }^{1}$ In Anlehnung an PPT (s. Chomsky 1981, Fanselow/Felix 1993:79-80, Stechow/Sternefeld 1988:230) wird das Pronomen es als Expletivum genannt, sobald es nicht im Sinne eines persönlichen Pronomens verwendet wird, d.h. sobald es kein Argument darstellt. Es ist ein semantisch leeres Element und bedeutet einen arbiträren, d.h. einen beliebigen Bezugspunkt. Daher entspricht der Begriff vom expletiven es mehreren Termini in anderen grammatischen Modellen. Zum Beispiel 
Bei den Expletiva handelt es sich um ein Quasiargument-es, das kasusmarkiert und merkmalsspezifisch ist, und um ein echtes Expletivum, das merkmalslos ist. Im Polnischen als eine pro-drop-Sprache ${ }^{2}$ wird an dieser Stelle von einem Argument-pro, Quasiargument-pro und einem expletiven pro ausgegangen.

\section{Die Satzstruktur und Checking-Konfigurationen}

In Anlehnung an Chomsky (1995, 2000) wird der Aufbau von Satzstrukturen durch drei syntaktische Operationen konstituiert: die Operation Merge, die zwei Elemente zu einem komplexen syntaktischen Objekt verbindet, die Operation Agree (Agreement), die Kongruenz und Kasus überprüft, und die Operation Move $\alpha$, die eine syntaktische Beziehung zwischen den einzelnen Konstituenten herstellt. $^{3}$ Die syntaktischen Bewegungsoperationen (Move $\alpha$ ) sowie die Operation der Verkettung (Merge) sind durch derivationale Notwendigkeiten, d. h. durch morphologische Merkmale und die Notwendigkeit ihrer Überprüfung (Checken) motiviert (vgl. Grewendorf 2002:156-158). ${ }^{4}$

Die funktionalen Köpfe, bei denen die Überprüfung erfolgt, enthalten Flexionsmerkmale, die mit den Flexionsaffixen assoziiert werden (s. Chomsky 1993:12f.). ${ }^{5}$

unterscheidet Engel (2009) in seiner dependenziellen Verbgrammatik zwischen es als expletives Element, es als fixes es oder auch es als Korrelat. Eisenberg (1986) bezeichnet es als „uneigentliches Subjekt", „Scheinsubjekt“, oder „formales Subjekt“. Bei Helbig (1991:88-90) handelt es sich jeweils um ein ,formales Subjekt". Duden (1984) bezeichnet es als Expletiv und weist auf drei seiner Funktionen hin: es als semantisch leerer Aktant bzw. semantisch leeres Subjekt, oder unpersönliches es; es als Korrelat, das auf einen nachgestellten Satz verweist; es als Platzhalter, das verschwindet, wenn ein beliebiges anderes Satzglied vor das finite Verb tritt.

2 Als pro-drop-Eigenschaft wird der Unterschied zwischen den Sprachen in Bezug auf die Wahlmöglichkeit von leeren Subjekten aufgefasst. Sie betrifft grammatische Bedingungen für die Auslassung von Subjekten finiter Sätze. Werden diese Bedingungen erfüllt, dann wird der pro-drop-Parameter positiv belegt. Als Haupteffekt der positiven Belegung des pro-drop-Parameters kann das Subjekt des finiten Satzes mit einer leeren Kategorie pro ersetzt werden (s. Chomsky 1981, Fanselow/Felix 1993, Grewendorf 1988, Grewendorf/Hamm/Sternefeld 1996, Stechow/Sternefeld 1988). Mit Chomskys (1982) Hypothese entspricht pro einem phonetisch nicht realisierten Personalpronomen. So weist die leere Kategorie pro pronominale Eigenschaften auf. Die allgemeinen syntaktischen Eigenschaften des Pro-drop-Parameters in Bezug auf das Polnische werden ausführlich in Pilarski (2013) bedacht.

${ }^{3}$ Move ist komplexer als Merge oder Agree und findet statt, wenn eine einfache Operation nicht möglich ist (s. Chomsky 2000:101).

${ }^{4}$ Minimalistisches Programm setzt voraus, dass die lexikalischen Elemente ihre Flexionsmerkmale bereits im Lexikon besitzen und sie werden mit ihnen aus dem Lexikon ausgewählt und in die Derivation eingesetzt (Enumeration).

5 Im Sinne von Chomsky (1993:12f.) gehören die Merkmale der funktionalen Köpfe zu den so genannten nicht-interpretierbaren Merkmalen (s. auch Grewendorf 2002: Kap. 6), Schmidt (1995: Kap. 1. 3. 3. 4.), die nach der Überprüfung getilgt werden. In Anlehnung an Chomsky (1993) kann 
Mit der Beobachtung, dass die Finitheit eines Satzes (die Merkmale von z.B. Tempus, Modus, ggf. Aspekt) mit dem Vorkommen von Kongruenzmerkmalen AGR(eement) wie Person, Numerus und ggf. Genus verbunden ist, bildete in PPT die Kategorie INFL (Inflection) den funktionalen Kopf. ${ }^{6}$

Im MP (s. Chomsky 2000) wurden die funktionalen Kategorien in Bezug auf die Frage entwickelt, ob sie sich durch morphosyntaktische Merkmale bestimmen lassen. Chomsky (2000:102) nimmt folgende funktionale Kategorien an: C (Satztyp/Force: deklarativ, interrogativ etc.), T (Tempus) und $v$ (der Kopf von transitiven Konstruktionen). Weiterhin betrachtet Chomsky (2008:143) die Phrase C als Kürzel für den Satzbereich, den Rizzi (1997) als linke Peripherie bezeichnet. TP konstituiert die temporale Struktur. Die Phrase $v$ wird mit der komplexen Argumentstruktur (Transitivität, Kausativität) assoziiert (s. auch Grohmann 2009). Sie konstituiert die verbale Struktur. VP enthält verfügbare NPs/DPs und thematische Rollen. Dies führt zur folgenden Konzeption der Satzstruktur:

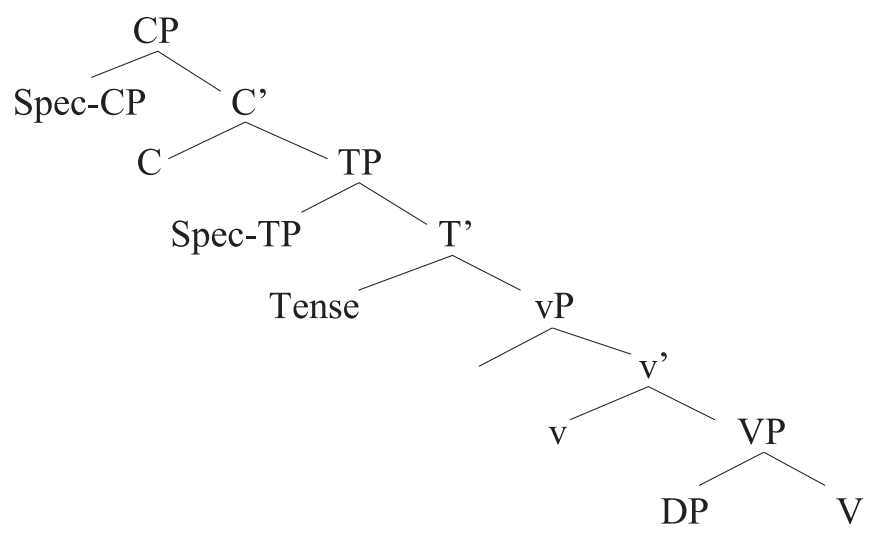

Rizzi (1997) analysiert die linke Satzperipherie und integriert Topik und Fokus in die Syntax. Er postuliert (Rizzi 2004: 6) folgende Köpfe im CP-Bereich: Illokution oder Satztyp (Force), Finitheit (Fin) wie Tempus und Modus. Zwischen Force

im Prinzip jeder funktionale Kopf als Überprüfer auftreten. Während die funktionalen Köpfe nur überprüfende Köpfe sind, enthalten die lexikalischen Köpfe nur zu überprüfende Merkmale. Nach Chomsky (1993) stellt sich die funktionale Kategorie als Vermittler zwischen lexikalischen Kategorien und deren Merkmalen dar. Wenn die Merkmale von lexikalischem Element und funktionalem Kopf übereinstimmen, dann werden die Merkmale des funktionalen Kopfes getilgt und als Effekt der Tilgung kommen morphologische Exponenten vor.

${ }^{6}$ Die INFL-Kategorie ist eine eigenständige und strukturaufbauende Kategorie, die die Kongruenzmerkmale auf die ganze funktionale Phrase überträgt (projiziert). Sie dehnt sich zu einem komplexen syntaktischen Gebilde aus (vgl. Fanselow/Felix 1993:55 - 56, Grewendorf 1995:125 - 126). Daher betrachtet man den Satz (im engeren Sinne) als eine Projektion von INFL. 
und Fin wird der Fokus platziert. Rizzi (1997) nimmt die folgende Einteilung in die vorgeschlagenen Projektionen:

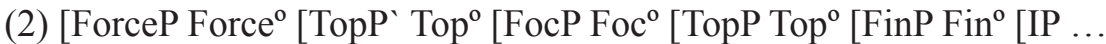

Nach Belletti (2004:16) wird der Fokus als funktionale Kategorie (Belletti 1999:13) von den Topik-Projizierungen eingerahmt. FokP (Fokus) und vP sind funktionale Projektionen (s. Belletti 1999, 2004, Belletti et al. 2007), in deren Spezifikator-Positionen der Nominativ zugewiesen wird ${ }^{7}$ und Kongruenzmerkmale überprüft werden (Agree in phi-features). ${ }^{8}$

TopP (Topik) und FokP (Fokus) sind miteinander korreliert. ${ }^{9}$ Bewegungen von Phrasen in eine entsprechende Position haben Konsequenzen für die semantische Interpretation (LF).

Parallel zur minimalistischen Reduzierung der funktionalen Kategorien entwickeln sich in generativen Arbeiten Tendenzen zur detaillierten Erfassung von Satzstrukturen, die als kartographische Ansätze (cartographic approach) benannt wurden. ${ }^{10}$ Die Komplexität kartographischer Darstellungen sollte aber in Bezug auf Einfachheit generativer Mechanismen im Minimalistischen Programm nicht als widersprüchlich, sondern als ergänzend und erläuternd betrachtet werden (s. Cinque/Rizzi 2008:49). Eine wichtige Ergänzung stellt in diesem Zusammenhang die Aufspaltung des INFL-Knotens dar, die von Pollock (1989) bei der Diagnose der systematischen Differenzierungen im Französischen und Englischen in Bezug auf die Syntax von Satznegation, Fragen, Adverbien, Quantoren und Quantifizierung vorgeschlagen wurde. Es wurden von ihm $A G R$ - und T(Tense/Tempus)-Köpfe der IP-Projektionen angenommen. Die Kasus- und Kongruenzmerkmale werden in der lokalen Checking-Domäne, d.h. in der Spec-AGRKopf-Relation überprüft (Chomsky 1995, Grewendorf 2002). ${ }^{11}$

7 Das Kasusmerkmal von NPs ist der sogenannte side effect einer existierenden Agree-Operation (s. Chomsky 2000).

${ }^{8}$ Die Bewegung in die Position, wo Agree stattfinden kann, wird durch das sogenannte EPP ausgelöst (s. Chomsky 2000).

9 Es gibt verschiedene Definitionen sowie verschiedene Typen von Topik und Fokus. Einen Überblick dafür liefert zum Beispiel Krifka (2006). Generell ist Topik das Element, worüber ausgesagt wird, und Fokus das Element, das eine neue Information bringt. Jedenfalls gibt es dazu verschiedene Stellungnahmen (s. u.a. Reinhart 1981, Reis 1999, Frey 2006). Durch Relationen von Topik kommt der Informationswert eines Satzes zum Ausdruck. Dazu gibt es verschiedene Konzepte in Bezug auf den Untersuchungsgegenstand (z.B. für die germanischen Sprachen u.a. Abraham 1992, 2005, für das Jiddische u.a. Prince 1989) und die Untersuchungsperspektive wie kartographische Konzepte von Belletti $(1999,2004)$ und Rizzi $(1997,2004)$.

10 Einen Überblick dazu liefert zum Beispiel Shlonsky (2010).

11 Nach Chomsky (1991) wurde AGR durch AGRs (Agreement-Subjekt) und AGRo (AgreementObjekt) mit ihren jeweiligen Projektionen ersetzt. Ein konzeptuelles Argument für die Ausdifferenzierung der Kongruenzphrase in AGR-Objekt-Phrase und AGR-Subjekt-Phrase stellte Chomsky 
(3) $\left[\right.$ AGRP $\left[\right.$ Spec- $\mathrm{I}^{\circ}$ Person, Numerus, Genus, / Nominativ AGR' $\left[\right.$ AGR ${ }^{\circ}$ Person, Numerus, Genus, / Nominativ V']]]

Während Cinque (2002) im Rahmen kartographischer Analysen bei der AGRPhrase bleibt, gibt es sie in späteren Publikationen von Chomsky (2000) und anderen Forschern (s. Belletti 2004, Rizzi 2004) nicht mehr. Agreement wird als eine in-situ-Operation aufgefasst. Sie ist aber für jegliche Bewegungsoperationen entscheidend, weil sie nur in bestimmten Konfigurationen sattfinden kann.

Für diesen Artikel sind die Merkmale des AGR-Kopfes insofern relevant, als er semantische Eigenschaften und Lizenzierungsbedingungen für die Subjekte bestimmt.

Geht man auf die Aufspaltung der IP zurück, dann gibt es nach Mohr (2005a, 2005b) mindestens zwei Subjektpositionen in der aufgespaltenen IP, nämlich SpecTP, die den Nominativ überprüft und SpecRefP, die für die Definitheit/Spezifizität verantwortlich ist. Definite Subjekt-DPs müssen sich bis nach SpecRefP bewegen. ${ }^{12}$

(4) $\left[\mathrm{CP}\left[\mathrm{C}^{\prime}\left[\mathrm{C}^{\circ}\left[\mathrm{IP}\left[\mathrm{RefP}<\mathrm{SpecRefP}><\mathrm{AGR}>\left[\mathrm{TP}\left[\mathrm{T}^{\prime}<\mathrm{SpecTP}>\left[\mathrm{T}^{\circ}[\mathrm{VP}\right.\right.\right.\right.\right.\right.\right.\right.$ $\left.\left.\left.\left.\left.\left.\left.\left.\left[\mathrm{V}^{\circ} \ldots\right][]\right]\right]\right]\right]\right]\right]\right]\right]$

Belletti (2004:17) nimmt weiterhin an, dass CP und IP die gleiche Strukturierung haben. Die linke Satzperipherie (CP) hat ihre Entsprechung in IP, die als Satz-Interne-Peripherie bezeichnet wird, d.h. sie hat eine Fokusprojektion, die von Topikprojektionen umgeben ist. Es wird hier die Idee von Belletti (1999) übernommen, dass Nominativzuweisung und Merkmalüberprüfung in der Relation Spec-Kopf von FokP erfolgen (s. Mecner 2016), was zur Fokussierung einer Subjekt-DP führt. Wie Mecner (2016) anhand der Analyse von jiddischen Sätzen zeigt, ist im Falle der Kasuszuweisung und Merkmalüberprüfung im tiefen FokP-Bereich der Fokusbereich der C-Domäne nicht aktiviert. Verfügbar bleibt eine nichtakzentuierte Top-Projektion. Integriert man die Annahme von Mecner (2016) in die Topund Fok-Projektionen im jiddischen Satz, wird für die weitere Untersuchung das folgende Schema angenommen:

(1991:424) anhand der Evidenzdaten von verschiedenen Sprachen, dass nicht nur Subjekte, sondern auch Objekte mit dem Verb kongruieren, dar.

12 Für eine SpecTP und SpecRefP argumentieren Mohr (2005a) mit dem Extended Projection Principle (EPP) und New Extension Condition und Diesing (1992) mit dem Mapping-Prinzip. Dieses besagt, dass ein definites Argument sich an der CI-Schnittstelle außerhalb der VP-Schale befinden muss. Dafür nimmt Diesing (1992) die SpecRefP und SpecTP. Vorher argumentieren auch Bobaljik/Jonas 1996 zum Isländischen, McCloskey 1996 zum Irischen, Kiss 1996 zum Englischen, oder Cardinaletti (1994) dafür, dass es (mindestens) zwei Subjektpositionen innerhalb der INFLProjektionen geben muss. 
(5)

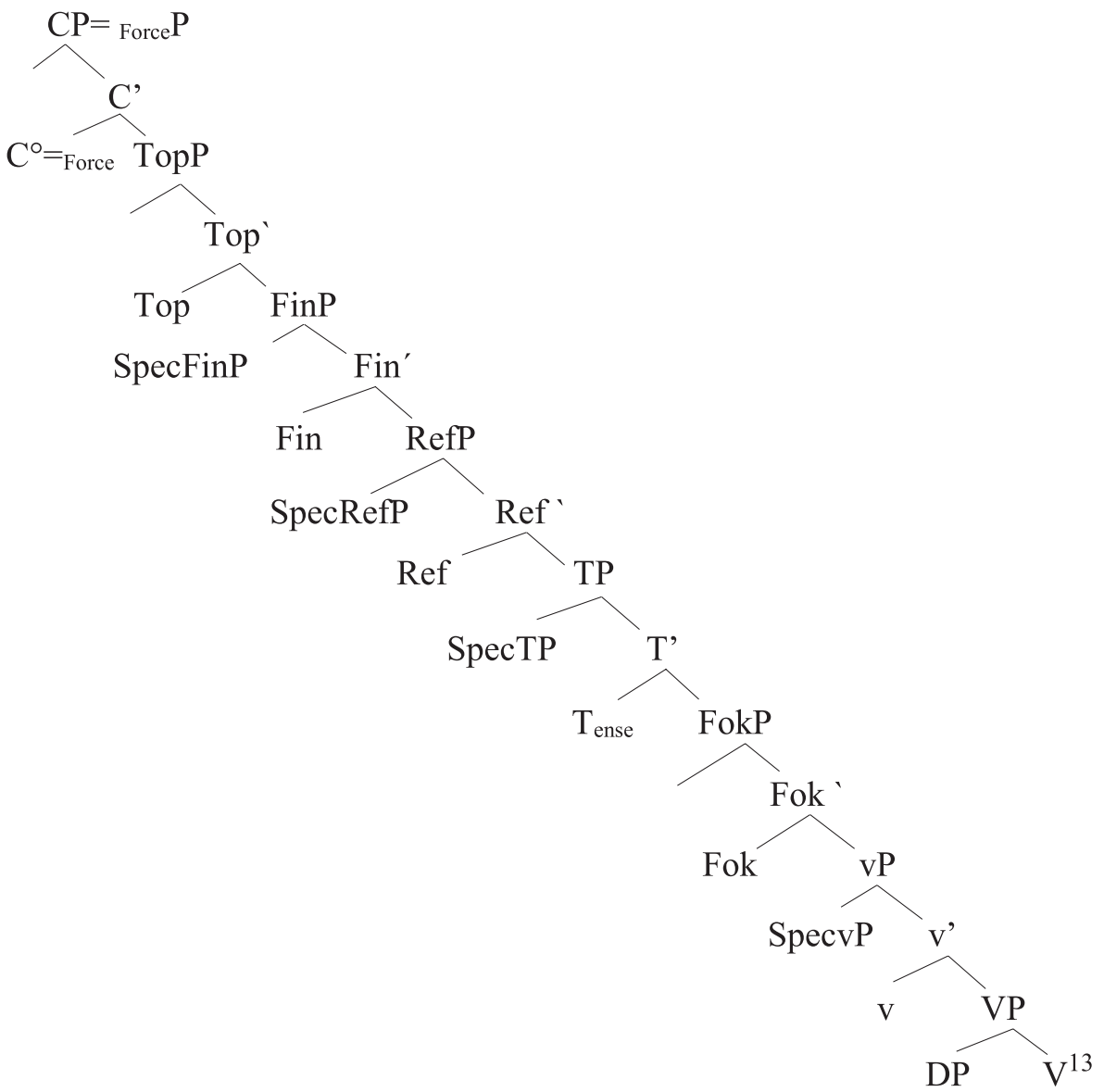

\section{Bestimmung der syntaktischen Struktur durch die Theta-Theorie}

\subsection{Argumentstruktur}

Nach dem Isomorphieprinzip (vgl. Schmidt 1995:102-104) ist die syntaktische Basisstruktur eines Satzes mit seiner lexikalisch-relationalen Struktur identisch. Mögliche reflektierte lexikalisch-thematische Relationen und ihre Zulassung in entsprechenden Konfigurationen bestimmt die Thetatheorie (s. Fanselow/Felix 1993:84, Schmidt 1995:54). Sie verbindet die logische Feststellung eines Arguments mit dessen struktureller Markierung. ${ }^{13}$

13 Die Theta-Rollen, wie z. B. Agens oder Patiens werden im Deutschen und im Polnischen als DPs realisiert, während z.B. die Theta-Rolle Instrument im Deutschen als Präpositionalphrase mit der Präposition mit und im Polnischen sowohl als Präpositionalphrase als auch als DP erscheint (vgl. Fanselow/Felix 1993:84). 
$\mathrm{Zu}$ den Argumenten gehören die nominalen Ausdrücke, die im Rahmen der Struktur, in der sie auftreten, referentielle Eigenschaften besitzen (vgl. Fanselow/ Felix 1993:79, Stechow/Sternefeld 1988:261). Die Argumente sind Träger der einzelnen Theta-Rollen und sie bilden mit dem Prädikat eine Konstituente. ${ }^{14}$ Das Thetakriterium besagt, dass jede Theta-Rolle genau einem einzigen Argument zugewiesen werden muss und umgekehrt, jedes der geforderten Argumente genau eine Theta-Rolle erhalten muss (vgl. Fanselow/Felix 1993:83).

Nach dem universellen Projektionsprinzip (vgl. Fanselow/Felix 1993:132, Stechow/Sternefeld 1988:295, Grewendorf 1995:121-122) wird dabei erzwungen, dass die im Lexikon festgelegte Argumentstruktur eines lexikalischen Elements auf jeder syntaktischen Repräsentationsebene erhalten bleibt. Daraus ergeben sich auch syntaktische Konsequenzen für die kategoriale Abstimmung zwischen der VP und der Subjekt-DP. Wird dem Subjekt eine Theta-Rolle zugewiesen, dann wird es durch eine lexikalische DP ausgedrückt.

(6) Ein Mädchen (es) hat, verträumt aus dem Fenster geschaut.

Eyn meydl (es) hot, farkholemt, aroysgekukt fun fentster.

Dziewczyna (ona), rozmarzona, patrzyła przez okno.

In den dargestellten Sätzen hat das Subjekt (ein Mädchen / eyn meydl / dziewczyna) die Theta-Rolle Agens. ${ }^{15}$ Nach Backer/Bobalijk (2002) stellt Agens immer ein externes Argument dar. ${ }^{16}$ Es steht auch im Theta-Raster hierarchisch höher als die internen Argumente. Nach Williams (1981, 1994) entspricht das externe Argument dem Subjekt und ist als Kopf der gesamten Argumentstruktur zu betrachten. Nach Mohr (2005a) werden Subjekte in SpecvP basisgeneriert. Weiterhin nimmt Mohr (2005a) an, dass eine vP immer vorhanden ist, auch wenn es sich um unakkusativische Verben handelt.

Nach Alexiadou/Anagnostopoulou (2001) geschieht eine Kasuszuweisung in der TP. Daher müssen die Argumente die VP verlassen, um ihren Kasus in der TP bekommen zu können. Gemäß dem EPP bei Mohr (2005a) sowie dem Mapping Prinzip bei Diesing (1992) wird der Kasus des Subjekts in der Spec-AGRs oder

14 Theta-Rollen sind die in der Bedeutung der Prädikate verankerten semantischen Rollen. Die Rollenkonstituierung ergibt sich indirekt aus den inhärenten semantischen Merkmalen der Prädikate. Grundlegend für den Entwurf eines Rolleninventars ist somit eine semantische Subklassifizierung der Prädikate (vgl. Järventausta 1991: Kap. 3.2.1.)

15 Die Zahl der Argumente eines Prädikats ist im mentalen Lexikon gespeichert, und die lexikalische Semantik eines Prädikats legt fest, welche Typen von Argumenten es verlangt. Das ThetaRaster ist ein morphosyntaktisches Merkmal. Die Konsequenz ist eine Unterscheidung zweier Arten von Argumenten. Diese bezieht sich auf die Argumentstellen eines logischen Prädikats und deren Realisierung in der Syntax. Je nachdem, ob ein Argument innerhalb oder außerhalb einer maximalen Projektion XP realisiert wird, werden syntaktische externe und interne Argumente unterschieden (vgl. Backer/Bobaljik 2002, Williams 1981).

${ }^{16}$ Liegt kein Agens, sondern ein Experiencer vor, dann ist dieser das externe Argument. Alle anderen sind die internen Argumente (vgl. Backer/Bobaljik 2002). 
SpecTP überprüft. ${ }^{17}$ Das Subjekt (ein Mädchen / eyn meydl / dziewczyna) wird daher aus der vP bewegt und nach TP angehoben. In Anlehnung an Mohr (2005a) und Diesing (1992) wird weiterhin das [+spezifisch]-Merkmal in der SpecRefP überprüft und anschließend nach SpecFinP angehoben. Die Anhebung des finiten Verbs bzw. Auxiliars nach FinP ist nach Belletti/Bennati/Sorace (2007), Mohr (2005b), Diesing (1992) in V-2-Stellung motiviert. Die satzinitiale XP besetzt in diesem Fall eine Spezifikatorposition in der aufgespaltenen CP, d.h. eine TopP oder SpecFinP.

(7) [FinP ein Mädchen hat / eyn meydl hot / dziewczyna patrzyta $[\operatorname{RefP}<$ ein Mädchen / eyn meydl / dziewczyna $><$ hat / hot / patrzyta $>$ [TP [vP <ein Mädchen / eyn meydl / dziewczyna > v [VP < aus dem Fenster / fun fentster / przez okno > geschaut / aroysgekukt]]]]]

\subsection{Es-Markierung in der Theta-Theorie}

Die Distribution und Referenzbestimmung des Subjekts in einem Satz ergibt sich aus dem Kasusfilter, das besagt, dass jedem Argument ein Kasus zugewiesen wird (vgl. Chomsky 1995:312, Fanselow/Felix 1993:78-79). Wird dem Subjekt keine Thetarolle zugewiesen, darf ein echtes Expletivum nur als beliebiger Bezugspunkt und lexikalische Füllung, nicht jedoch als Argument in der Subjektposition stehen (vgl. Fanselow/Felix 1993:78). ${ }^{18}$ In diesem Fall ist das Pronomen es merkmalslos, kann kein Kasusmerkmal überprüfen und nur in der Position eingefügt werden, in der es auch sichtbar auftritt, d.h. es kann sich nicht bewegen. Nach Mohr (2005b) tritt das echte Expletivum in V2-Sprachen nur in SpecFinP auf. ${ }^{19}$

In Anlehnung an Fanselow (1987:216) treten im Deutschen auch Verben mit den so genannten Quasi-Thetarollen auf. Sie zeichnen sich dadurch aus, dass sie unter funktionaler aber nicht willkürlicher Kontrolle auftreten, wie z.B. Manchmal regnet es, ohne (e) zu hageln. (Fanselow 1987:216). ${ }^{20}$ Bei diesen Verben liegt bezüglich ihrer es-Subjekte die Kontrollfähigkeit vor, d. h. das leere Subjekt

17 Für eine ausführliche Diskussion dazu siehe Chomsky (2008), Diesing (1992) und Mohr (2005a).

18 Das Expletivum es besetzt bei bestimmten Verben die Subjektposition, aber es darf nicht in den Komplementstrukturen auftreten (vgl. Fanselow/Felix 1993:80).

19 Die Annahme der SpecFinP für das expletive es beruht auf der Überlegung, dass das expletive es nicht als notwendige Subjektmarkierung, sondern als Eigenschaft einer V-2-Sprache anzusehen ist, so dass seine Einsetzung nicht mit dem Subjekt selbst, sondern mit der Besetzung der Vorfeldposition zu tun hat. Als Vorfeldpositionen gelten in der aufgespaltenen CP die SpecTopP und SpecFinP. Da es sich beim expletiven es um keine topikalisierte oder kontrastakzenttragende Phrase handelt, darf es nicht die SpecTopP besetzen (vgl. Belletti/Bennati/Sorace 2007, Mohr 2005a, Diesing 1992).

20 Die Ansetzung der leeren Kategorie PRO als implizites Subjekt im infiniten Komplementsatz wird in der PPT durch das Kontrollphänomen gefordert. Die Kontrolle wird durch die Koindizierungsregel bezeichnet, die zwischen PRO und einer Kontrolleur-NP des Matrixsatzes erfolgt. Nach PRO-Theorem ist PRO Träger einer Theta-Rolle. Seine Einsetzung durch Referenz impliziert somit, dass PRO eine Theta-Rolle hat, die seinem Bezugselement gleich ist. 
(e) in der infiniten Einbettung bezieht sich auf das Matrix-Subjekt. Die Referenz von $e$ zur DP im Matrixsatz unterscheidet das expletive es als Quasiargument vom echten expletiven es, das keine Kontrollfähigkeit besitzt, d.h. das „leere Subjekt“ tritt im infiniten Teil ohne Referenz zu einer DP im Matrixsatz auf (vgl. Grewendorf 1988:140, 162-163, Stechow/Sternefeld 1988:305-308). Beim expletiven es erweisen sich die Sätze als ungrammatisch: *Gestern hungerte es mich, anstatt mich zu frieren. (Fanselow 1987:216).

\subsection{Pro-Gehalt}

Die syntaktisch-semantische Interpretation eines Satzes findet in verschiedenen Sprachen einen unterschiedlichen artikulatorisch-phonologischen Ausdruck. So kann im Polnischen in der 1. und 2. Person in finiten Sätzen das Subjekt phonetisch nicht markiert werden. Die Theta-Rolle, die ein Verb an das Subjekt vergibt, darf aber nicht aufgelöst werden, sondern sie muss durch ein bestimmtes Element in der Subjektposition erkennbar gemacht werden. Dies übernimmt in den finiten Sätzen im Polnischen als eine pro-drop-Sprache die leere Kategorie $p r o .{ }^{21}$

Die Zuweisung einer Thetarolle ermöglicht die Interpretation von pro, sobald pro formal durch INFL lizenziert und identifiziert wird (vgl. Fortmann 1996:156, Huang 1984:552), Upyong (1995:80). ${ }^{22}$

21 Die theoretischen Voraussetzungen für die Kategorie pro sind durch die Charakterisierung der funktionalen Kategorie INFL in Bezug auf Flexionseigenschaften der Agreement (Kongruenz)-Elemente verfügbar. Die INFL enthält einen finiten Merkmalskomplex, der mit seinem Spezifizierer, d.h. dem Subjekt in Person, Numerus und ggf. Genus übereinstimmt. Dank INFL muss das Subjekt nicht vorkommen und trotzdem ist dieses erkennbar. Außer der Kategorie pro gibt es auch andere leere, d.h. nicht-lexikalisierte Kategorien in den Satzstrukturen. Die leeren Kategorien werden als derivierte Leerstellen oder als basisgenerierte leere Elemente interpretiert. Sie stehen in einem direkten Zusammenhang mit dem Projektionsprinzip, dem Theta-Kriterium, dem generalisierten Empty-Category-Prinzip (ECP), der Rektions- und Bindungstheorie sowie der Kontrolltheorie. Das Problem der Interpretation leerer Subjekte in Bezug auf die einzelnen Lizenzierungsbedingungen wird in diesem Beitrag nicht diskutiert.

22 Die Wiedererkennung des pro-Gehalts setzt voraus, dass ein hinreichend reiches Flexionssystem in einer Sprache vorhanden ist, das durch starke Merkmale gekennzeichnet ist (vgl. Fanselow/Felix 1993:211 - 218, Fortmann 1996:156, Stechow/Sternefeld 1988:294-295). So zeigen die polnischen Sätze, dass trotz fehlender phonetischer Markierung des pronominalen Subjekts, es durch morphologische Exponenten am Verb angezeigt werden kann:

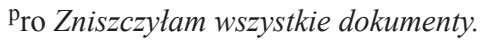

[pro vernichtete +1 . Person,. Singular, Femininum alle Dokumente]

Ich/er/sie/es vernichtete alle Dokumente.

Entsprechend der PPT-Terminologie (vgl. Speas 1994:185) verfügt das Polnische über die sogenannte starke Kongruenz. Die starke Kongruenz involviert die starken Merkmale, die nach dem Checking-Prozess an funktionalen Köpfen markiert werden. Somit findet die Besetzung der SpecI-Position durch pro in finiten Sätzen im Polnischen ihre Rechtfertigung. Die starke INFL im Polnischen erlaubt nämlich aufgrund ihrer grammatischen Informationen das ausgelassene Subjekt 
Wenn INFL nicht in der Lage ist, den AGR-Merkmalsgehalt von pro festzulegen, stellt pro kein Argument dar. Folglich wird dem pro eine arbiträre Interpretation und arbiträre Thetarolle zugesprochen. Man spricht in diesem Fall auch von einem arbiträren, d.h. expletivem pro, das als beliebiger Bezugspunkt in der Subjektposition steht (vgl. Fanselow/Felix 1993:78). ${ }^{23}$

Je nachdem in welchem Grad das AGR-Element in INFL fähig ist, AGR-Merkmale zu spezifizieren, führt Rizzi (1986) eine Hierarchie der pro-Elemente ein. Die pro-Elemente werden in expletives pro, Quasiargument-pro und Argument-pro eingeteilt (vgl. Fortmann 1996:162).

\section{Quasi-Thetarolle und Subjektmarkierung}

\subsection{Quasiargument-es im Deutschen}

Nach Chomskys (1981) Auffassung verfügen die Wetterverben über eine Quasi-Thetarolle, die von einem pronominalen Element getragen wird. Somit müsste vP ein Quasiargument (im Folgenden: Q-Arg) als Subjekt in der Spec-vP-Position lizenzieren. Nach Mecner (2016) werden anhand seiner ausführlichen Diskussion über Subjektüberprüfung in jiddischen Sätzen werden unakkusativische Verben in der funktionalen Projektion vP überprüft.

(8) $\left[\mathrm{vp}_{\mathrm{vP}}<(\mathrm{Q}-\mathrm{Arg})\right.$ es $>\left[{ }_{\mathrm{v}}<\right.$ regnet $\left.\left.>[\mathrm{vP}]\right]\right]$

In Anlehnung an Mohr (2005) tragen weiterhin die Quasiargumente sowohl ein Kasusmerkmal (Nominativ) als auch das Merkmal [+spezifisch]. Daher werden sie in SpecvP basisgeneriert und müssen sich in der aufgespaltenen IP zwecks Kasusüberprüfung durch SpecTP nach SpecRefP bewegen, wo sie das Definitheitsmerkmal überprüfen.

zu erschließen (vgl. Pilarski 2002:49-50,110-111). Die pronominalen Subjekte in Sprachen mit weniger reichem Flexionssystem können nicht weggelassen werden. Neben der Identifizierung vom pro durch die AGR-Merkmale in INFL kann im polnischen Satz für eine Identifizierung durch c-kommandierende DP postuliert werden. Als adäquat für Distribution von pro lässt sich an dieser Stelle die Theorie der generalisierten Kontrolle (RGK) von Huang $(1984,1989)$ auffassen. Eine gründliche Auseinandersetzung zu diesem Thema findet man u.a. in Upyong (1995) am Beispiel von chinesischen Sätzen; s. auch Pilarski (2003). Das Problem der Interpretation leerer Subjekte in Bezug auf RGK wird in diesem Artikel nicht diskutiert.

23 Die Bedingung für eine formale Lizenzierung ist aber, dass pro hier kein Argument ist. Wird pro als Nonargument generiert, dann braucht es die Arg-Merkmale nicht vollständig zu spezifizieren. Dies findet gerade im Deutschen beim unpersönlichen Passiv seine Rechtfertigung. In diesem Fall handelt es sich ausdrücklich um das expletive pro. 
(9) ${ }_{\text {FinP }}$ es regnet $\left[{ }_{\mathrm{RefP}}<e s><\right.$ regnet $>{ }_{\mathrm{TTP}_{\mathrm{P}}<e s}><$ regnet $>{ }_{\mathrm{VP}}<e s>{ }_{\mathrm{v}}<$ regnet $>\left[{ }_{\mathrm{VP}}\right.$ $<$ regnet $>$ heute $>]]]]$

Daraus resultiert, dass das expletive es als Q-Arg in einem Satz obligatorisch ist (vgl. Mohr 2005). Dafür findet man im Deutschen eine positive Evidenz. Das expletive es tritt in Haupt- (10a) und Nebensätzen (10b) auf, auch wenn eine andere $\mathrm{XP}$ in satzinitialer Position steht.

(10) (a) Es regnet.

(b) ... weil es heute regnet. (Fanselow 1987:216).

...*weil heute regnet.

(c) Heute regnet es.

In einem eingebetteten Satz wie in (10b) kann man annehmen, dass das expletive es als Subjekt in SpecvP eingesetzt wird. Die Bewegung des finiten Verbs nach T erfordert die Erzeugung von SpecTP, damit das Kasusmerkmal überprüft werden kann (s. Alexiadou/Anagnostopoulou 2001, Mohr 2005a). ${ }^{24}$ Folglich bewegt sich das Subjekt aus der vP nach SpecTP und anschließend wird es nach SpecRefP angehoben, um das [+spezifisch]-Merkmal zu checken. Zusätzlich wird der Komplementierer in Force-CP eingesetzt.

(11) ${ }_{\text {ForceP }}$ weil $\left[{ }_{\text {FinP }}\right.$ es regnet $\left[{ }_{\text {RefP }}<e s><\right.$ regnet $>\left[{ }_{\mathrm{T}} \mathrm{P}<\right.$ es $><$ regnet $>\left[{ }_{\mathrm{V}} \mathrm{P}<e s>\mathrm{V}\right.$ $<$ regnet $>[\mathrm{VP} \mathrm{v}<$ regnet $><$ heute $>]]]]]$

\subsection{Quasiargument-pro im Polnischen}

Im Polnischen, das eine pro-drop-Sprache ist, findet keine es-Markierung statt. Sollten aber die Wetterverben ein Q-Arg selegieren, dann müsste das Q-Arg-pro ${ }^{25}$ dessen Trägerfunktion übernehmen. Die Interpretation vom pro erfolgt nämlich aufgrund der Zuweisung von AGR-Merkmalen via Thetarollenzuweisung (s. Rizzi 1986, Fortmann 1996:158), d.h., wenn dem Subjekt eine Theta-Rolle zugewiesen wird, muss es durch AGR-Merkmale in INFL angezeigt werden, und umgekehrt sollte es möglich sein, die pro-Kategorie durch AGR-Merkmale zu identifizieren,

${ }^{24}$ Gemäß EPP/Extended Projection Principle und der ausführlichen Diskussion dazu in Mohr 2005 b muss die Kasuszuweisung in Spec von AGRs oder in Spec von T geschehen. Zwar gelten bei Chomsky (2000:102) nur C und $v$ als Derivationsphasen, diese sind allerdings mit der Annahme der Kasusüberprüfung in der TP nicht widersprüchlich, denn Chomsky nimmt TP in der CTv-System an.

25 Nach dem universellen erweiterten Projektionsprinzip (vgl. Grewendorf 1995:121 - 122, Stechow/ Sternefeld 1988:295) hat nämlich jeder Satz ein Subjekt. 
so ist die Determinierung ihres Gehalts erforderlich. In Bezug auf diese Überlegung könnte man für einen Satz wie Pada. „Es regnet“ eine folgende Struktur annehmen:

$$
\left[{ }_{\mathrm{vP}}<(\mathrm{Q}-\mathrm{Arg}) \text { pro }>\left[{ }_{\mathrm{v}}<\text { pada }, \text {,regnet } ">[\mathrm{vP}<\text { pada , regnet" }>]\right]\right]
$$

Die Untersuchung der Wetterverben im Präsens und Präteritum (13a, 13b) zeigt jedoch, dass das Wetterverb im Polnischen eine Default-Form (DF) ${ }^{26}$ aufweist. Sie beinhaltet zwar ein T(empus)-Merkmal, dieses muss aber nicht sofort, sondern erst durch Instanziierung der Zeitrelation auf LF (Logischer Form) überprüft werden. ${ }^{27}$ Im Polnischen bedeutet die Default-Form eine unveränderliche Flexionsform der 3. Person Singular Neutrum [3. P., Sg., Neut.]. Dieser Verbform entspricht nach Grewendorf (2002:170) keine DP als Subjekt. ${ }^{28}$

(13) (a) Pada [3. Pers., Sing., Neut.].

$\left[\right.$ Regnet $\left._{\text {(Default-Form) }} \varnothing\right]$

Es regnet.

(b) Wczoraj padało [3. Pers., Sing., Neut.].

[Gestern regnete $($ Default-Form) $\varnothing]$

Gestern regnete es.

Um das EPP (erweitertes Projektionsprinzip) nicht zu verletzen, wonach jeder Satz ein Subjekt hat (vgl. Grewendorf 1995:121-122, Stechow/Sternefeld 1988:295), könnte man in den Sätzen (13a, 13b) ein formales Funktionssubjekt annehmen. Dieses zeichnet sich jedoch dadurch aus, dass es semantisch leer ist (vgl. Grewendorf 2002:170). Folglich fungiert es nicht als Anzeiger für eine vollständige Flexion am Verb. ${ }^{29}$ Wie in Pilarski $(2013,2015)$ dargestellt wird, verfügt das Polnische über das expletive pro, das als nicht morphologisch markiertes Funktionssubjekt gilt. Das Funktionssubjekt kommt in einigen Negationssätzen, in den Sätzen mit Quantoren und in einigen unpersönlichen Konstruktionen vor. ${ }^{30}$

26 Die syntaktische AGR-Kategorie am Verb ist hier als defektive Form zu betrachten, weil sie keine Unterscheidung in Person, Numerus und Genus des Subjekts berücksichtigt.

27 Nach Chomsky (1993) ist die DF durch schwache Merkmale gekennzeichnet, so dass sie erst auf der Ebene LF gecheckt wird. Für eine Überprüfung der Merkmale von DF auf LF argumentiert auch Preminger (2014).

28 Es gibt nämlich kein lexikalisches Element als Nominativsubjekt, das eine bestimmte Flexion am Verb verlangt. Daher wird dem Verb eine Default-Form zugeteilt.

29 Das expletive pro weist in vielen Konstruktionen des Polnischen eine arbiträre Referenz zu.

30 Die folgenden dargestellten Sätze zeichnet aus, dass hier keine ersichtliche Übereinstimmung des finiten Verbs mit dem Subjekt stattfindet.

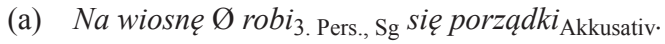

[Zum Frühling Ø macht sich Ordnungen]

Zum Frühling mach man Ordnung.

(b) $W$ domu nie było 3 . Pers., Sg. $\varnothing ~ o j c a_{\text {Genitiv }}$. 
Der Satzstruktur nach entspricht das expletive pro in Wetterverbkonstruktionen dem echten expletiven es im Jiddischen. Die V-2-Stellung im Jiddischen verlangt jedoch die lexikalische Vorfeldbesetzung, so dass im Jiddischen das Pronomen es am Satzanfang erscheint.

\subsection{Die Merkmale des Jiddischen}

Betrachtet man die Sätze im Jiddischen, wird die Annahme von Quasiargumenten in den Konstruktionen mit den Wetterverben in Frage gestellt. Die Verteilungsfakten des Jiddischen zeigen, dass das expletive es nicht obligatorisch ist, sondern nur am Satzanfang stehen kann (vgl. 14a mit 14b). In Nebensätzen tritt das expletive es auf, wenn es direkt auf den Komplementierer folgt (vgl. 14c mit 14d).

(14) (a) es hot nekhtn geregnt.

Es hat gestern geregnet.

(b) ?nekhtn hot es geregnt.

Gestern hat es geregnet.

(c) ... vayl s'iz ${ }^{31}$ nekhtn gegangen a regn

.... weil es gestern geregnet hat.

(d) ... ? vayl nekhtn iz es gegangen a regn.

... weil es gestern geregnet hat.

In Anlehnung an Mohrs (2005) Merkmalsbedingungen für es-Interpretationen und aus der Beobachtung, dass das echte expletive es nur in satzinitialer Position grammatisch ist, könnte man von einem echten Expletivum in den jiddischen

\footnotetext{
Zu Hause gab es keinen Vater.

(c) $W$ piosenkarce $z_{\text {akochato }}$ [3. P., Sg., Neut.] się $\varnothing$ pięciu $_{\text {Genitiv }}$ fanów $_{\text {Genitiv. }}$

In die Sängerin verliebten sich fünf Fans.
}

Es ist ersichtlich, dass der DF vom Verb kein lexikalisches Subjekt entspricht. Es gibt nämlich kein lexikalisches Element als Nominativsubjekt, das eine bestimmte Flexion am Verb verlangt. So ist anzunehmen, dass der Nominativ im Polnischen außer einer lexikalischen NP in der Subjektposition auch ein Kennzeichnen für das formale Funktionssubjekt ist. Aufgrund der Feststellung, dass das Polnische zu den pro-drop-Sprachen gehört, wonach die Subjektleerstellen auf der PF-Ebene (Phonetische Form) zulässig sind, darf das formale Funktionssubjekt nicht morphologisch markiert werden. Daher kann Default Value in engerem Sinne als leere Kategorie pro expl $_{1}$ interpretiert werden. Nach Grewendorf (2002:170) ist es als eine vorangestellte Eingabe anzusehen, die keinen eigenen Wert hat, sondern einen so genannten Default Value (im Folgenden DV) darstellt. Der Begriff Default Value wird folglich für das Funktionselement verwendet, das eine leere Kategorie darstellt, d.h. für eine leere Kategorie pro expl $_{1}$ im Polnischen. Im Satz (a) lässt das pro $_{\text {expl }}$ Menschen und in den Sätzen (b)-(c) die Genitivmarkierte DP (des Arztes, der Schüler) als „logische“ Subjekte auf der semantischen Ebene interpretieren.

31 ,se“ und ,s" " sind die phonologischen Varianten von „es“. 
Wetterverbkonstruktionen ausgehen. Diese Annahme wird durch die V-2-Stellung im Jiddischen unterstützt. ${ }^{32}$

Als Argument für das echte Expletivum statt Q-Arg-es gilt auch die Tatsache, dass das es-Element mit einer anderen XP alternieren kann, sobald diese topikalisiert wird (15a, 15b). Diese Erscheinung betrifft sowohl Haupt- als auch Nebensätze des Jiddischen.

(15) (a) nekhtn hot geregnt.

[Gestern hat geregnet.]

Gestern hat es geregnet.

(b) ... vayl nekhtn iz gegangen a regn.

[... weil gestern geregnet hat.]

... weil es gestern geregnet hat.

Darüber hinaus lassen die jiddischen Sätze die Einfügung einer DP nach dem Wetterverb zu, die mit diesem Verb in Kongruenzbeziehung steht. ${ }^{33}$

(16) s'iz nekhtn gegangen a regn. (Mohr 2005).

[Es ist gestern niedergegangen ein Regen]

Es ist gestern ein Regenschauer niedergegangen./Es hat gestern geregnet. (Mohr 2005).

Aufgrund der AGR-Merkmale kann man annehmen, dass die DP a regn „ein Regen" das Subjekt des Wetterverbs darstellt. Da in der thetamarkierten Position nur ein Argument stehen darf, müsste in diesem Fall das Pronomen es als Q-Arg getilgt werden. Nach dem Theta-Kriterium schließen sich das Q-Arg-es und DP gegenseitig aus. Aus dem Satz (16) ist jedoch ersichtlich, dass das Pronomen es unberührt bleibt. Es ist somit als echtes Expletivum anzusehen, das keine Theta-Rolle trägt und folglich parallel zum Subjekt-DP erscheinen kann. Seine Tilgung erfolgt durch die Topikalisierungsoperation wie in (17):

(17) nekhtn iz gegangen a regn.

[gestern ist niedergegangen ein Regen]

Gestern hat es geregnet.

32 Im Deutschen und Jiddischen als Verb-Zweit-Sprache eröffnet das Verb ein Vorfeld, das die Regelungen in Bezug auf die lexikalische Füllung bringt.

33 Mohr (2005) zeigt, dass das Verb regnen im Jiddischen unterschiedlich interpretiert werden kann. Im Satz wie haynt regnt es konfeti. 'Heute regnet's Konfetti.' argumentiert sie für ein Quasi-Argument und transitiven Gebrauch des Wetterverbs. Die Existenz eines Quasiarguments lizenziert nach Mohr (2005) in diesem Satz eine akkusative DP. Das Verb regnen kann auch periphrastisch ausgedrückt werden. In diesem Fall platziert Mohr (2005) die DP in der SpecvP-Position, wo sie als Subjekt anzusehen ist und wo sie ihr Kasusmerkmal überprüft. 


\section{4. Ähnlichkeit mit dem Polnischen}

Vergleicht man die jiddische Konstruktion mit ihrer polnischen Entsprechung wie in (18), weist das Polnische eine Ähnlichkeit mit dem Jiddischen in Bezug auf die Subjektmarkierung auf.

(18) (a) Wczoraj padat deszcz.

[Gestern regnete Regen]

Gestern regnete es.

(b) Wczoraj padat śnieg.

[Gestern regnete Schnee]

Gestern schneite es.

Die Verteilungsfakten des Polnischen lassen die nach dem Wetterverb stehende DP als Subjekt-DP interpretieren. Die DP deszcz „der Regen“ bzw. śnieg „der Schnee" weist AGR-Merkmale auf. Sie ist nominativmarkiert und entspricht der Verbendung der 3. Pers. Sing. Maskulinum (19a). Da im Polnischen das pro $_{\text {expl }}$ als Entsprechung des echten expletiven es im Deutschen merkmalsfrei ist, findet im Polnischen eine vollständige AGR-Markierung erst dann statt, wenn die Subjektposition lexikalisch durch eine DP besetzt wird. Fehlt die genannte DP im Satz, dann weist das Wetterverb im Polnischen eine DF auf (19b).

(19) (a) Wczoraj padat [3. Pers., Sing., Mask.] $\operatorname{deszcz}$ [3. Pers., Sing., Mask.].

[Gestern regnete Regen]

Gestern regnete es.

(b) Wczoraj padało [3. Pers., Sing., Neut.].

[Gestern regnete $($ Default-Form) $\varnothing]$

Gestern regnete es.

\section{Checking-Prozess der DP}

\subsection{Endposition der DP in Wetterverbsätzen}

Die jiddischen und polnischen Wetterverbsätze weisen eine Gemeinsamkeit in Bezug auf die Stellung der eingefügten nominativmarkierten DPs auf. In beiden Sprachen handelt es sich nämlich um eine postverbale Stellung dieser DPs (20a, 20b). Es ist sichtbar, dass die finale Position der Subjekt-DP eine neutrale Position ist, d.h. die Subjekt-DP trägt in dieser Position den Normalakzent. ${ }^{34}$ Die Stel-

34 Die postverbale Stellung der Subjekt-DP beeinflusst dabei die satzinitiale Position, so dass hier der Kontrastakzent nicht mehr möglich ist. Es muss an dieser Stelle von Konfigurationen unterschieden werden, die bei kausativen transitiven Verben mit der finalen Subjektposition entstehen, 
lung des Subjekts beeinflusst auch die Informationsstruktur des Wetterverbsatzes. Steht das Subjekt am Satzende, ist es fokussiert, d.h. die Subjekt-DP ist in der Fokusprojektion der I-Domäne platziert. Sollte die jeweilige Subjekt-DP dagegen die präverbale Position einnehmen, wird sie akzentuiert (20c, 20d). Aus informationstruktureller Sicht ist sie in der topikalisierten Phrase in der Fokusprojektion der C-Domäne platziert.

(20) (a) Wczoraj padat deszcz.

[Gestern regnete Regen]

(b) nekhtn iz gegangen a regn. (Mohr 2005).

[Gestern ist niedergegangen ein Regen]

s'iz nekhtn gegangen a regn. (Mohr 2005).

[Es ist gestern niedergegangen ein Regen]

(c) Deszcz' wczoraj padat.

[Regen gestern regnete]

Ein Regen ist gestern niedergegangen.

(d) a regn' iz nekhtn gegangen.

Ein Regen ist gestern niedergegangen.

Im Gegensatz zu den Konstruktionen mit Wetterverben ist das Subjekt Topik in vielen anderen Sätzen, wo es auch nicht akzentuiert wird (s. Mecner 2016). ${ }^{35}$

(21) der tate Gunter Viner hot farmogt groyse gesheftn mit briln

Der Vater Gunter Winer besaß große Brillengeschäfte. (Forverts 05.03.2013, s. Mecner 2016).

\subsection{Checken in der Fokusposition}

An dieser Stelle ist zu fragen, wie die finale DP (im Polnischen deszcz, im Jiddischen a regn) ihren Kasus überprüft.

In neueren Ansätzen (vgl. Belletti 1999, 2004, Belletti et al. 2007) wird postuliert, dass die Kasusüberprüfung durch Fokus lizenziert wird. Der Nominativ wäre in diesem Fall eine unmarkierte Form overter, d.h. phonologisch marki-

wo entweder Topik oder Fokus akzentuiert werden (vgl. Mecner 2016) sowie von Konfigurationen, bei denen ein kontrastiver sprecherintentionaler Akzent gesetzt wird.

35 Mecner (2006) weist darauf hin, dass es im Jiddischen auch Konstruktionen mit Subjekt in der Fokus-Position gibt, wie z.B. fun untern dakh efent zikh a kleyn fensterl 'Von unterm Dach öffnet sich ein kleines Fenster.' (vgl. Peretz, ed. 1920). Eine ausführliche Diskussion zu diesen Konstruktionen findet man in Mecner (2006). In diesem Artikel werden sie nicht einbezogen. Sie stellen aber wertvolle Tools dar für die funktionale Nutzung der Fokus-Phrase bei den Wetterverben im Jiddischen und Polnischen. 
erter DPs. Da für die Merkmalsüberprüfungen eine Spezifikator-Kopf-Beziehung notwendig ist, muss die finale DP in die SpecPosition von FokP angehoben werden, um den Nominativ zu bekommen und Kongruenzmerkmale zu überprüfen.

Daraus resultiert die Annahme, dass die besprochenen DPs in der vP vorerst keine Kasus tragen dürfen. Diese Annahme findet mit dem Konzept der Generalisierung von Burzio (1986) ${ }^{36}$ ihre Bestätigung. Nach ihr kann eine DP in der Komplementposition einen Akkusativ bekommen, wenn das Verb ein externes Argument (Agens) hat.

Betrachtet man die Wetterverbsätze im Jiddischen und Polnischen, wird in diesen Sätzen ersichtlich, dass die nominativmarkierten DPs (deszcz, a regn) keine Agens darstellen und es gibt keinen anderen Urheber des Geschehens. ${ }^{37}$ So liegt eine implizite Annahme vor, dass eine DP in der Kopf-Komplement-Relation (V DP) des jiddischen Wetterverbsatzes keinen Objektkasus zugewiesen bekommt. Es gibt in der Struktur keine andere DP, die als Agens interpretiert wird und das Verb ist nicht kausativ (vgl. Mecner 2016). Folglich ist die SpecvP leer. ${ }^{38}$

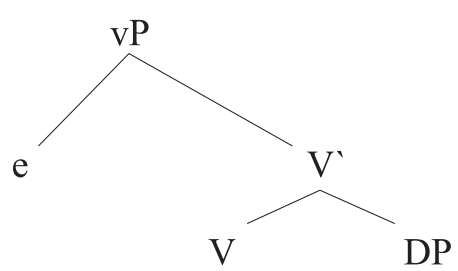

Als weitere Konsequenz kann in der Konfiguration (V DP) das Merkmal Kasus [CAUSE] nicht aktiviert werden. Die V-Anhebung erreicht in diesem Fall in den $\nu$ P-Bereich das Merkmal [-CAUSE] und sie kann keinen Akkusativ lizenzieren. ${ }^{39}$

\footnotetext{
36 Der Versuch, das Phänomen der oben dargestellten Konfiguration im Jiddischen zu erklären, geht auch auf das Konzept der Unakkusativität (Perlmutter 1978) zurück.

37 In beiden Fällen sind die Verben als Vorgangsverben zu interpretieren.

38 Wie oben dargestellt, trägt das Pronomen es in diesem Fall keine Theta-Rolle.

39 Wie Mohr (2005) am Satzbeispiel aus dem Jiddischen wie haynt regnt es konfeti. „Heute regnet's Konfetti.“ zeigt, lizenziert nach Burzios Generalisierung (1986) die Existenz eines Quasiarguments in Wetterverb-Konstruktionen eine akkusative DP bei transitivem Gebrauch des Wetterverbs. Im dargestellten Beispiel wird es als Quasiargument analysiert, das in SpecvP eingesetzt und dann nach SpecTP angehoben wird. In Bezug auf diese Überlegung findet die Annahme einer Dekausativierung bei Wetterverben ihre Rechtfertigung. Eine ausführliche Untersuchung zum Konzept der Dekausativierung im Jiddischen findet man in Mecner (2016).
} 
Die vP kann mit folgendem Schema dargestellt werden:

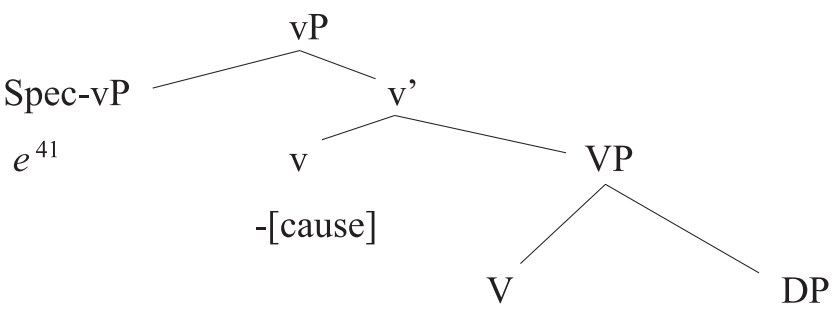

In der funktionalen Projektion vP werden unakkusativische Verben [-CAUSE] entsprechend überprüft. Die tief liegende VP enthält eine verfügbare Komplement-NP mit der zugewiesenen thematischen Rolle Thema. Jedenfalls wird sie nicht akkusativmarkiert. Der Spezifizierer von vP ist leer.

Das finite Verb (im Jiddischen in diesem Fall das Auxiliar ${ }^{40} i z$ ) V wird in Fokusposition angehoben. Darauf folgt die Bewegung der DP (deszcz, a regn 'der Regen') in die SpecPosition von FokP, woraus Checking in der Spec-Kopf-Relation resultiert. ${ }^{41}$ Weiterhin wird das finite Verb in die T-Position (Tempus) bewegt. Wie Mecner (2016) anhand seiner ausführlichen Diskussion über die jiddischen Sätze mit Extraposition der Subjekte annimmt, ist das Expletiv bereits in der VP enthalten. Mecner (2016) spricht an dieser Stelle von der Möglichkeit einer Aktivierung bzw. Deaktivierung des expletiven es. Es wird aktiviert, wenn die DP in die SpecPosition von FokP angehoben wird und kein frei verfügbares Element in die C-Domäne bewegt wird. ${ }^{42}$ Jedenfalls erfolgt seine Deaktivierung, wenn eine andere Phrase vor dem Finitum platziert ist, d.h. bei der Anhebung der DP in die SpecPosition von TP oder bei der Besetzung der TopPosition durch eine XP. ${ }^{43}$

Die Satzstruktur mit dem Wetterverb und der Subjekt-DP in der Fokusposition am Satzende kann man in Anlehnung an Belletti (1999, 2004, Belletti et al. 2007) mit folgendem Schema darstellen:

\footnotetext{
40 Das Jiddische bildet die Vergangenheit nur mit dem Perfekt.

41 Mohr (2005) schlägt vor, dass die DP in die SpecFocP nicht angehoben, sondern gleichgesetzt wird, weil sie auch ohne Bewegung linear in der Fokusposition am Satzende steht.

42 In der V-2-Sprache wird ein Vorfeld vor dem Verb eröffnet, das die Regelungen in Bezug auf die lexikalische Füllung bringt. Die Bestätigung dafür liefern im Jiddischen die Nebensätze mit ihrer symmetrischen Position des finiten Verbs. So erfolgt im Gegensatz zum Deutschen eine es-Markierung auch in einem Nebensatz, wenn die Position vor dem Verb leer ist.

43 In deklarativen Sätzen des Jiddischen ist auch eine Verbvoranstellung möglich. Diese steht parallel zu den nicht akzentuierten XPs und den Expletiva: hot zikh gefirt tsvishn em un dem foter a milkhome [hat sich geführt zwischen ihm und dem Vater ein Krieg] 'Es wurde zwischen ihm und dem Vater ein Krieg geführt.' (Bimko 1921, s. Mecner 2016). Nach Mecner (2016) wird bei der Verbvoranstellung das Expletiv ebenfalls deaktiviert.
} 
(24)

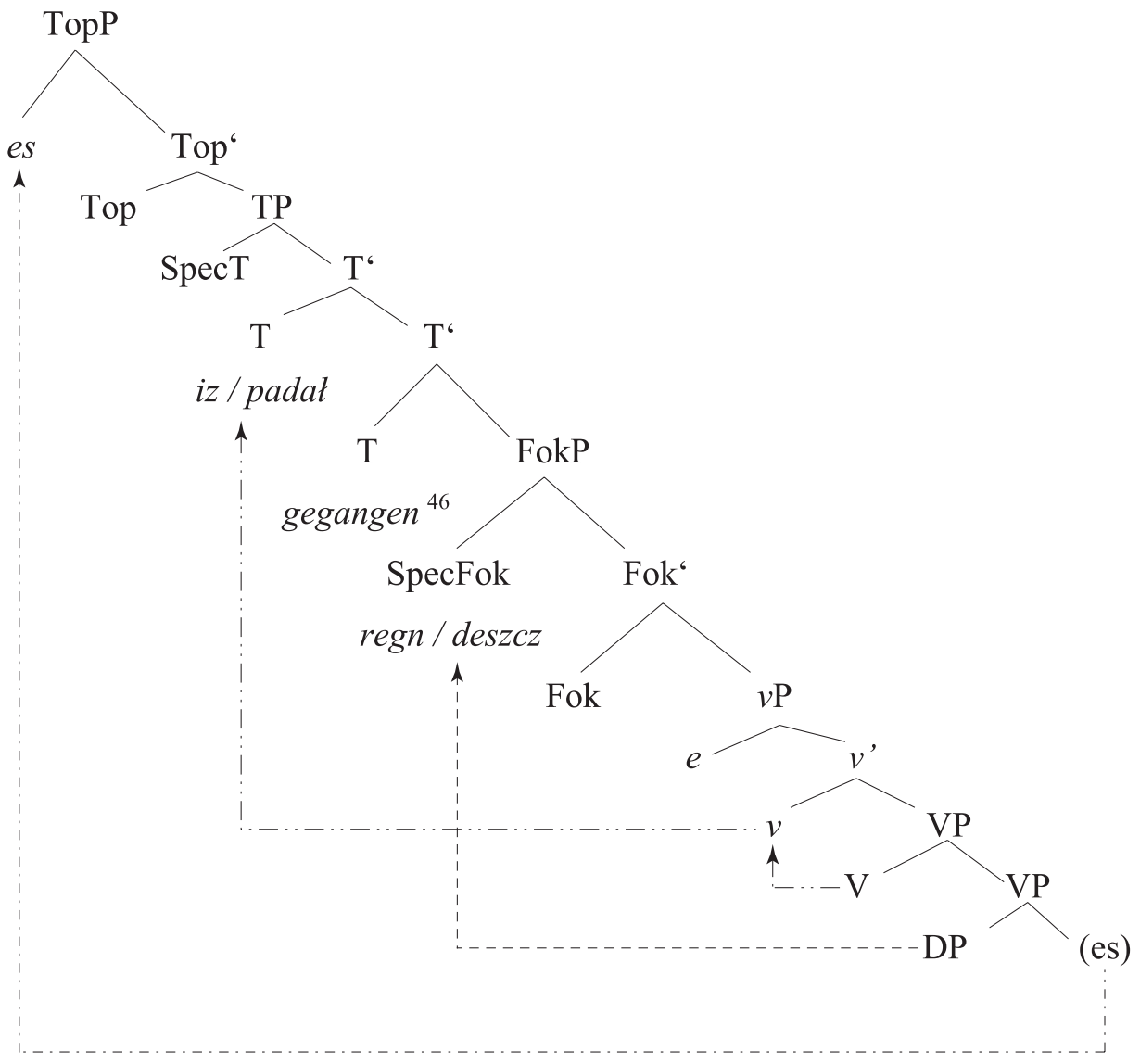

\section{Schlussfolgerungen}

In Hinsicht auf die Untersuchung der Wetterverbsätze weisen die syntaktischen Eigenschaften des Jiddischen Gemeinsamkeiten mit dem Polnischen und dem Deutschen als seine Kontaktsprachen auf. Wie im Deutschen verfügt das Jiddische über das expletive Subjekt es, das das Polnische gar nicht kennt. Bei Wetterverben des Deutschen handelt es sich um ein Quasiargument es. Seine lexikalische Realisierung ist in allen Konfigurationen obligatorisch. Da die semantische Interpretation (LF) von einer syntaktischen Konfiguration abhängig ist, muss der phonologische Exponent es als Quasiargument für das Deutsche interpretiert werden. 
Durch den Vergleich mit dem Polnischen zeigt aber das Jiddische, dass das expletive es keine Theta-Rolle trägt. Daraus resultiert, dass es als Nonargument interpretiert wird, und somit dem echtem Expletivum entspricht. Dieses ist mit dem expletiven pro im Polnischen zu vergleichen. Im Polnischen findet das expletive Element auf phonologischer Ebene keinen lexikalischen Ausdruck.

Als echtes Expletivum ist das Pronomen es in den jiddischen Sätzen mit Wetterverben nicht obligatorisch und es ist nur in satzinitialer Position grammatisch korrekt. Darüber hinaus kann es sowohl in Haupt- als auch in Nebensätzen mit einer topikalisierten XP alternieren. Das Pronomen es trägt das Merkmal [+spezifisch] nicht. Es wird eingesetzt, wenn die TP oder TopP unbesetzt bleiben. Sonst wird seine lexikalische Realisierung deaktiviert.

Die Annahme von es als Expletivum im Jiddischen und pro als expletives pro im Polnischen kann mit der Tatsache bestätigt werden, dass die Wetterverbsätze eine mit dem Verb kongruierende nominativmarkierte DP in postverbaler Stellung zulassen. Diese ist aber nicht als Agens zu interpretieren, sondern sie trägt die thematische Rolle Thema. Ihre Merkmale werden in der SpecPosition der funktionalen Kategorie FokP überprüft. Wenn die Kasuszuweisung und Merkmalüberprüfung im FokP-Bereich erfolgt, sind keine weiteren Bewegungen der DP notwendig. Dadurch ist die gesamte Operation sparsamer. Der lokale SpecKopf-Bereich von FokP ist nämlich die nächstliegende Projektion. Daraus resultiert auch die Endposition der nominativmarkierten DP. Der Effekt der Fokussierung vom Subjekt korreliert mit der Nutzung von TopP im C-Bereich. In dieser Position sind eine adverbiale PP oder ein Expletivum frei verfügbar. Beide tragen dabei den Satznormalakzent.

\section{Literaturverzeichnis}

ABRAHAM Werner, 1992, Überlegungen zur satzgrammatischen Begründung der Diskursfunktionen Thema und Rhema, in: Folia Linguistica 26/1-2, S. 198-231.

ABRAHAM Werner, 1995, ${ }^{2} 2005$, Deutsche Syntax im Sprachenvergleich: Grundlegung einer typologischen Syntax des Deutschen. 2., verbesserte und erweiterte Auflage, Tübingen.

AleXiadou Artemis / AnAgnostopoulou Elena, 2001, The Subject-in-situ Generalization and the Role of Case in Driving Computations, in: Linguistic Inquiry 32, S. $193-231$.

Belletti Adriana, 1999, Inversion as focalization and related questions, in: Catalan Working Papers in Linguistics 7, S. $9-45$.

Belletti Adriana, 2004, Aspects of the Low IP Area, in: Rizzi L. (Hrsg.), The Structure of CP and IP. The Cartography of Syntactic Structures, Vol. 2, New York, S. 16-51.

BELLETTI Adriana / BENNATI Elisa / SORACE Antonella, 2007, Theoretical and developmental issues in the syntax of subjects: Evidence from near-native Italian, in: Natural language \& linguistic theory 25 , S. $657-689$.

BAKER Mark / BOBALJIK Jonathan, 2002, Introduction to Morphology, Ms. Rutgers University and McGill University. 
BobaLJIK Jonathan / JoNAS Dianne, 1996, Subject Positions and the Role of TP, in: Linguistic Inquiry 27, S. $195-236$.

BURZIO Luigi, 1986, Italian Syntax, Dordrecht.

CARDinaletti Anna, 1994, Subject Positions, in: GenGenP 2/1, S. $64-78$.

CHOMSKY Noam, 1981, Lectures on Government and Binding, The Pisa Lectures, Dordrecht.

CHOMSKY Noam, 1982, Some concepts and consequences of the Theory of Government and Binding, Cambridge, Mass./London.

ChOMSKY Noam, 1991, Some Notes on Economy of Derivation and Representation, in: Freidin R. (Hrsg.), Principles and Parameters in Generative Grammar, Cambridge/Mass./London, S. $417-454$.

ChOmsky Noam, 1993, A Minimalist Program for Linguistic Theory, in: Hale K./Keyser S.J. (Hrsg.), The View from Building 20. Essays in Linguistics in Honour of Sylvian Bromberger, Cambridge/Mass./London, S. 1-52.

ChOMSKY Noam / LASNIK Howard, 1993, A Theory of Principles and Parameters, in: Jacobs J./ Stechow A./Sternefeld W./Vennemann T. (Hrsg.), Syntax. Ein internationales Handbuch zeitgenössischer Forschung, Berlin, S. 506-569.

CHOMSKY Noam, 1995, The minimalist program, Cambridge/Mass./London.

CHOMsky Noam, 2000, Minimalist Inquiries: The Framework, in: Martin R./Michaels D./Uriagereka J. (Hrsg.), Cambridge/Mass./London, S. 89-156.

Chomsky Noam, 2008, On Phases, in: Peregrin O./Carlos N. et al. (Hrsg.), Foundational Issues in Linguistic Theory: Essays in Honor of Jean-Roger Vergnaud, Cambridge/Mass., S. 133-166.

CINQue Guglielmo, 2002, The Cartography of Syntactic Structures, Bd. 1, Oxford.

Cinque Guglielmo / Rizzi Luigi, 2008, The Cartography of Syntactic Structures, in: Studies in Linguistics. Working Papers 2, S. $42-58$.

DIESING Molly, 1992, Bare plural subjects and the derivation of logical representations, in: Linguistic Inquiry 23(3), S. 353-380.

DIESING Molly, 1997, Yiddish VP Order and the Typology of Object Movement in Germanic, in: Natural Language \& Linguistic Theory 15(2), S. 369-427.

DiesING Molly, 2005, The Upper Functional Domain in Yiddish, in: Abraham W. (Hrsg.), Focus on Germanic Typology, in: Studia Typologica 6, Berlin, S. 195-209.

Duden Bd. 4., 1959, ${ }^{4}$ 1984, Grammatik der deutschen Gegenwartssprache, Drosdowski G. et al. (Hrsg.), Mannheim/Leipzig/Wien/Zürich.

EISENBERG Peter, 1986, Grundriss der deutschen Grammatik, Stuttgart.

ENGEL Ulrich, 2004, ${ }^{2} 2009$, Deutsche Grammatik - Neubearbeitung, München.

FANSELOW Gisbert, 1987, Konfigurationalität. Untersuchungen zur Universalgrammatik am Beispiel des Deutschen, Tübingen.

FANSELOW Gisbert / FELIX Sascha, 1993, Sprachtheorie 2: Rektions- und Bindungstheorie, Tübingen.

FreY Werner, 2006, How to get an object-es into the German prefield, in: Brandt P./Fuss E. (Hrsg.), Form, Structure, and Grammar - A Festschrift Presented to Günther Grewendorf on Occasion of His 60th Birthday, Berlin, S. 159-185.

FORTMANN Christian, 1996, Konstituentenbewegung in der DP-Struktur. Zur funktionalen Analyse der Nominalphrase im Deutschen, Tübingen.

GREWENDORF Günther, 1988, Aspekte der deutschen Syntax. Eine Rektions-Bindungs-Analyse, Tübingen.

GREWENDORF Günther, 1995, Sprache als Organ - Sprache als Lebensform, Frankfurt am Main.

GREWENDORF Günther, 2002, Minimalistische Syntax, Tübingen/Basel.

GREWENDORF Günther / HAMM Fritz / STERNEFELD Wolfgang, 1987, ${ }^{8} 1996$, Sprachliches Wissen: Eine Einführung in moderne Theorien der grammatischen Beschreibung, Frankfurt am Main. 
GroHmanN Kleanthes K. (Hrsg.), 2009, Explorations of Phase Theory: Features and Arguments, Berlin.

HELBIG Gerhard, 1991, Deutsche Grammatik - Grundfragen und Abriss, München.

Holmberg Anders / PlATZACK Christer, 1990, The Rolle of AGR and Finiteness in Some European VO Languages, Paper presented at GLOW, Utrecht.

HUANG C.-T. James, 1984, On the distribution and reference of empty pronouns, in: Linguistic Inquiry 17, S. $501-557$.

HuANG C.-T. James, 1989, Pro-drop in Chinese: a generalized control theory, in: Jaeggli O./Safir K.J. (Hrsg.), Studies in Natural Language and Linguistic Theory: The null subject parameter, Dordrecht/Boston/London, S. 185-214.

JÄRVENTAUSTA Marja, 1991, Das Subjekt im Deutschen und im Finnischen: seine Formen und semantischen Rollen, Frankfurt am Main.

KIss Katalin É., 1996, Two Subject Positions in English, in: The Linguistic Review 13, S. 119-142.

KRIFKA Manfred, 2006, Basic Notions of Information Structure, in: Féry C./Fanselow G./Krifka M. (Hrsg.), Working Papers of the SFB632, Interdisciplinary Studies on Information Structure (ISIS) 6, Potsdam, S. $13-56$.

MCClOSKeY James, 1996, Subjects and Subject Positions in Irish, in: Borsley R.D./Roberts I. (Hrsg.), The Syntax of the Celtic Languages: A Comparative Perspective, Cambridge, S. $241-283$.

MECNER Pawel, 2016, Zur Korrelation zwischen satzfinaler Subjektposition, Verbgruppe und satzinitialer Position im Jiddischen, Manuskript.

MoHr Sabine, 2005a, Eine Antwort auf die Frage, wo dem Jiddischen der Kopf steht. GGS (Generative Grammatik des Südens), Tübingen, [http://ifla.uni-stuttgart.de/files/ggs_2005.pdf, Zugriff am 08.04.2014].

MoHR Sabine, 2005b, Clausal architecture and subjects positions: Impersonal con-structions in the Germanic languages, Amsterdam.

Perlmutter David M., 1978, Impersonal Passives and the Unaccusative Hypothesis. Proceedings of the 4th Annual Meeting of the Berkeley Linguistics Society, S. 157-190.

PILARSKI Anna, 2002, Die Operation „Merge“ im Verbalkomplex des Polnischen und des Deutschen, Frankfurt am Main.

PILARSKI Anna, 2003, Zur Pro-drop-Eigenschaft im Polnischen und im Deutschen. Kwartalnik Neofilologiczny 3, Warszawa, S. 355-371.

PILARSKI Anna, 2013, Das Nullsubjekt im Polnischen. Dependenzielle Verbgrammatik und Generative Transformationsgrammatik im Modellvergleich, München.

PILARSKI Anna, 2015, Die CP- und AGR-Struktur der VS-Folge im Polnischen, in: Wierzbicka M./ Golonka J. (Hrsg.), Grammatische Strukturen im Text und im Diskurs. Bd. 5, Rzeszów, S. $105-124$.

Pollock Jean-Yves, 1989, Verb Movement, Universal Grammar, and the Structure of IP, in: Linguistic Inquiry 20, Cambridge/Mass./London, S. 365-424.

PREMINGER Omer, 2014, Agreement and its failures. Linguistic Inquiry Monographs 68, Cambridge.

PRINCE Ellen, 1989, Yiddish wh-clauses, subject-postponing, and topicalization, in: Powers J./de Jong K. (Hrsg.), ESCOL 88. Columbus, S. $403-415$.

ReINHART Tanya, 1981, Pragmatics and linguistics: an analysis of sentence topics, in: Philosophica 27, S. $53-94$.

REIS Marga, 1999, On sentence types in German: an enquiry into the relationship between Grammar and Pragmatics, in: Interdisciplinary Journal for Germanic Linguistics and Semiotic Analysis, 4.2, S. $195-236$.

RIZZI Luigi, 1986, Null object in Italian and the Theory of pro, in: Linguistic Inquiry 17, Cambridge/ Mass./London, S. $501-557$. 
RizzI Luigi, 1997, The Fine Structure of the Left Periphery, in: Haegeman L. (Hrsg.), Elements of Grammar; Handbook in Generative Syntax, Dordrecht, S. 281 - 338.

RIzZI Luigi (Hrsg.), 2004, The Structure of CP and IP. The Cartography of Syntactic Structures, Vol. 2, New York.

SADOCK Jerrold, 1998, A Vestige of Verb Final Syntax in Yiddish, in: Monatshefte für deutschsprachige Literatur und Kultur 90, S. 220-226.

SANTORINI Beatrice, 1993a, The rate of phrase structure change in the history of Yiddish, in: Language Variation and Change 5, S. 257-283.

SANTORINI Beatrice,1993b, Das Jiddische als OV/VO-Sprache, in: Linguistische Berichte 123, S. $230-245$.

SCHMIDT Claudia Maria, 1995, Satzstruktur und Verbbewegung. Eine minimalistische Analyse zur internen Syntax der IP ( Inflection Phrase) im Deutschen, Tübingen.

ShlONSKY Ur, 2010, The Cartographic Enterprise in Syntax, in: Language and Linguistics Compass 4/6, S. $417-429$.

SPEAS Peggy, 1994, Null arguments in a theory of economy of projection, in: Benedicto E./Runner J. (Hrsg.), S. 179-208.

STECHOW Arnim / STERNEFELD Wolfgang, 1988, Bausteine syntaktischen Wissens. Ein Lehrbuch der generativen Grammatik, Opladen.

Travis Lisa, 1991, Parameters of Phrase Structure and Verb Second Phenomena, in: Freidin R. (Hrsg.), Principles and Parameters in Comparative Grammar, Cambridge, S. 339-364.

UPYONG Hong, 1995, Null-Subjekte im Erst- und Zweitspracherwerb des Deutschen, Tübingen.

VIKNER Sten, 2013, Verb Particles in Germanic SVO- and SOV-languages: Yiddish compared to Danish and German. Denmark: University of Aarhus, [http://www.hum.au.dk/engelsk/engsv/ handouts/vikner-ho-2013-Cambridge-particles.pdf, Zugriff am 07.05.2014.].

Wallenberg Joel C., 2013, Scrambling, LF, and Phrase Structure Change in Yiddish, in: Lingua 133, S. $289-318$.

WiLliams Edwin, 1981, Argument Structure and morphology, in: The Linguistic Review 1, S. $81-114$.

WiLLIAMS Edwin, 1994, Thematic structure in syntax, Cambridge/Mass.

\section{Quellenangaben}

Antologye fun deryidisher proze in Polyn tsvishn beyde velt-milkhomes (1914-1939), Nyu-York 1946 (= Bimko 1921, I. Bashevis Singer 1931).

Di verk fun I.L. Perets.Dritter Band. Nyu-York 1920. (= Peretz, ed. 1920).

Forverts 05.03.2013.

אַ פַינטעלע גוי פוֹ

http://yiddish.forward.com/articles/168333/non-jewish-identity/\#ixzz2Mn09mlpg.

MECNER Paweł, 2016, Zur Korrelation zwischen satzfinaler Subjektposition, Verbgruppe und satzinitialer Position im Jiddischen, Manuskript.

MoHR Sabine, 2005, Eine Antwort auf die Frage, wo dem Jiddischen der Kopf steht. GGS (Generative Grammatik des Südens), Tübingen, [http://ifla.uni-stuttgart.de/files/ggs_2005.pdf, Zugriff am 08.04.2014]. 